\title{
Exploring Structure and Dynamics of Biological Assemblies by Digital- Imaging Cryo-TEM
}

\author{
Dganit Danino* \\ *Technion, Department of Biotechnology and Food Engineering, Haifa, Israel, 32000.
}

Direct-imaging cryo-transmission electron microscopy (cryo-TEM) is a powerful tool for studying macromolecular assemblies in the 1 to $300 \mathrm{~nm}$ range, forming by biological complexes and molecules (viruses, lipids, proteins, DNA), and biological fluids (bile, lungs fluid). Until a few years ago technical difficulties limited the use of cryo-TEM primarily to 3D structural studies requiring high resolution. However, digital imaging and procedures we have developed specifically for morphological studies [Danino et al., 2001] allow now to readily and directly obtain fine structural details such as size, shape, internal ordering and coexistence of different structures, at $\sim 1 \mathrm{~nm}$ resolution. In addition, we can explore mechanisms of self-assembly, interactions between molecules, structural transitions, formation of transient structures during dynamic processes, and structure-function relationships in complex biological systems, and we can uncover fine assembly details hidden until recently.

Several examples are presented in the poster, one study is described here in detail.

Taxol is a most widely used drug in cancer chemotherapy, particularly effective in gynecological cancers. It is a concentrated solution containing Paclitaxel in Cremophore EL (polyoxyethylated castor oil, CrEL) and ethanol, that must be further diluted before administration. In $2 \%$ of treatments Taxol causes severe immediate hypersensitivity reactions that can be lethal. It has been suggested the hypersensitivity is caused by compliment (C) activation. In clinically relevant aqueous solutions of CrEL, small spheroidal micelles, 8-18 $\mathrm{nm}$ in diameter, are observed (A); The concentrations in these solutions exceeds the CMC by 400 to 1800 -fold, suggesting CrEL is infused in patients mainly in a micellar form. In Taxol samples the micelles coexist with micrometer long, crystalline, needle-like structures (B-D). Multimolecular aggregates, possibly lipoproteins found in human plasma are shown in (E). Upon dilution with CrEL and Taxol many larger aggregates (50-300 nm in diameter) are found, giving the impressions of oil droplets $(\mathrm{F})$, suggesting an interaction of the drug with 
plasma lipoproteins, We found CrEL micelles and crystalline structures in all clinically relevant solutions of Taxol. Apparently, self-aggregation of the drug components is induced by dilution in the infusion bag, which upon injection to the blood may lead to the severe hypersensitivity reactions due to complement (C) activation.

This study was done in collaboration with Yeshayahu Talmon, Carl Alving. Aba Priev, Janos Szebeni, Sandor Savay and Yechezkel Barenholz.
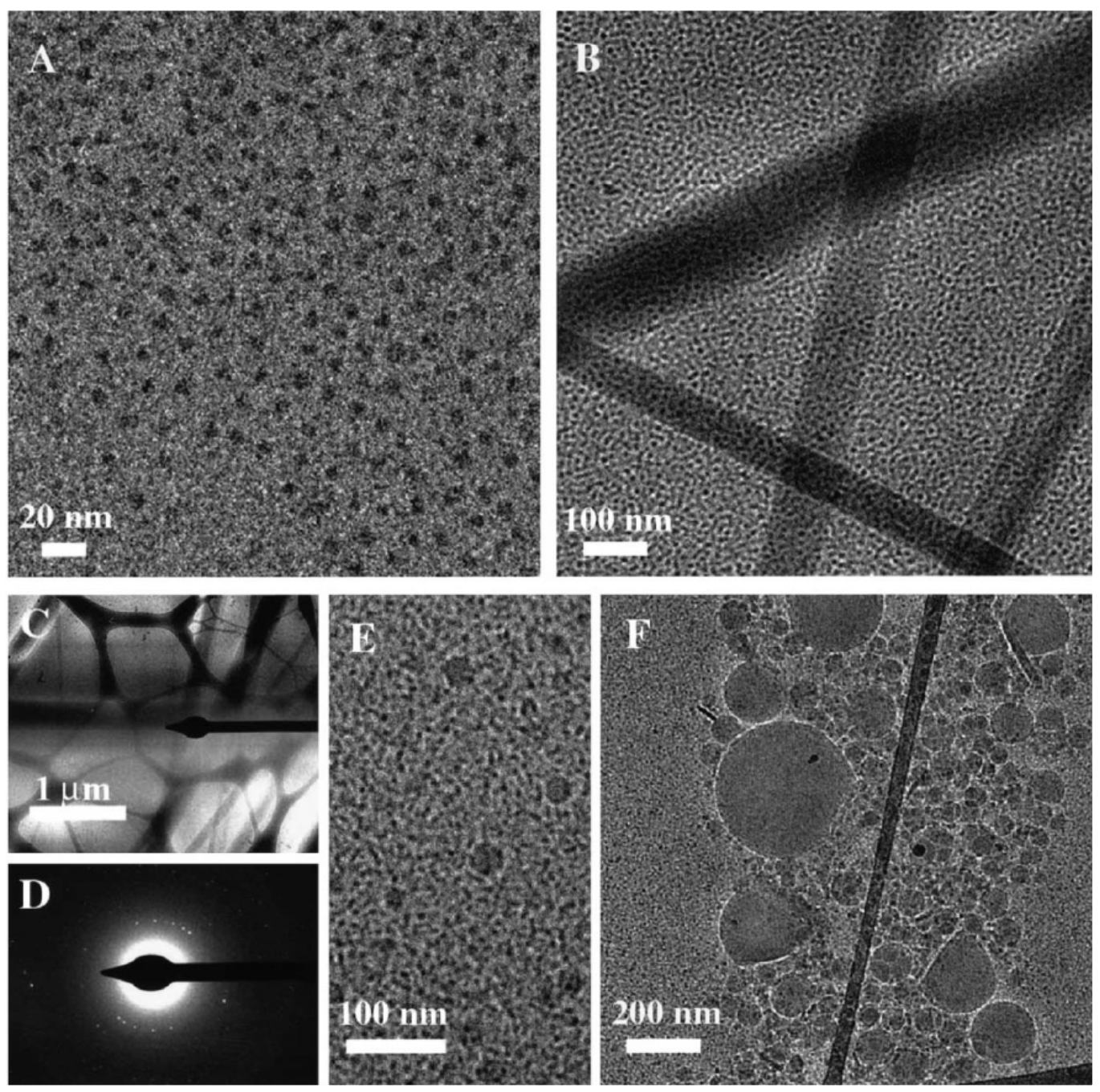

[1] Danino et al., Digital Cryogenic Transmission Electron Microscopy: an Advanced Tool for Direct Imaging of Complex Fluids, Colloids and Surfaces A: Physicochemical and Engineering Aspects 183, 113-122 (2001).

[2] Szebeni et al., Formation of Complement-Activating Particles in Aqueous Solutions of Taxol: Possible Role in Hypersensitivity Reactions Int Immunopharmacol 1(4), 721-735 (2001). 